Editorial

\title{
Antibacterial Quorum-Sensing Molecules as Long Lasting Antibacterial Drugs
}

\author{
${ }^{1}$ Ananya Pavuluri, ${ }^{2}$ Yan Zhang and ${ }^{3}$ Shanzhi Wang \\ ${ }^{1}$ New Rochelle High School, 256 Clove Road, New Rochelle, NY10801 \\ ${ }^{2}$ Eye Hospital, 2nd Teaching Hospital of Jilin University, Changchun, China 130000 \\ ${ }^{3}$ Department of Cell Biology, Albert Einstein College of Medicine, Yeshiva University, 1300 Morris Park Avenue, Bronx, NY \\ 10461, United States
}

\author{
Article history \\ Received 05-09-2014 \\ Revised 03-12-2014 \\ Accepted 16-01-2015 \\ Corresponding Author: \\ Yan Zhang \\ Eye Hospital, 2nd Teaching \\ Hospital of Jilin University, \\ Changchun, China 130000 \\ E-mail: zhangy66@jlu.edu.cn
}

\begin{abstract}
Antibacterial quorum-sensing molecules are a new type of antibacterial reagent that blocks bacterial communications and dampens virulence. These molecules are proposed to tackle the roots of drug resistance and to be long lasting, although more research should be done toward the effectiveness and resistance of these drugs.
\end{abstract}

What happens if an army is replaced by a motley crew in a battle? Most likely, the chance of winning the battle will be significantly compromised. Then, what is the chance of winning if the motley crew further disarms their weapons? When bacterial pathogens attack our defense system, they are a unified army with synchronized motility, biofilm formation and virulence (Miller and Bassler, 2001).

In another scenario, what if this army is surrounded by their enemies, who swear to kill every single one of them in the battlefield? Naturally, everyone of this army is going to fight fiercely under this survival pressure. However, what if they were told that none of them will die if they disperse and disarm themselves? During an infection, the application of antibiotics put the bacterial army under survival pressure, resulting in bacteria fighting backantibiotic resistance (Andersson and Hughes, 2010).

Thus, an ideal alternative to antibiotics should act to destroy the bacterial pathogen city, but not the bacteria. This leaves the bacteria to the immune system, resulting in significantly delayed or zero drug-resistance. Quorum Sensing (QS) is the process of inter-bacterial communication, which synchronizes bacterial behaviors and regulates pathogen city. QS benefits a bacterial community by causing the bacteria to act in unison, but it is usually not essential for bacterial survival or growth. Thus, in contrast to traditional antibiotics, inhibition of QS does not apply survival or selection pressure, only transforming a bacterial army into an unarmed motley crew. As such, QS Inhibitors (QSIs) are seen as potential long-lasting antibacterial drugs (Allen et al., 2014) and many patents of QSIs were published in the last few years (Jiang and Li, 2013).

However, different opinions are raised on whether QSIs can be long-lasting or resistance-proof. Indeed, QS inhibition places bacteria under the disadvantage of being vulnerable, raising the concern that bacteria may develop resistance to QSIs (Defoirdt et al., 2010), similar to the development of antibiotic resistance. This view is supported by a recent report which showed that the opportunistic pathogen Pseudomonas aeruginosa, whose QS system was extensively studied, rapidly developed resistance to QS inhibitor brominated furanone C-30 by mutations to increase efflux (Maeda et al., 2012).

The results highlight the concerns of developing QSI-resistance. However, alternative explanations can support the theory that QSIs' effects can be long lasting. In the study highlighted above, random transposon mutagenesis was used to speed up the generation of resistance, which may not happen naturally. In addition, the bacteria have been cultured in an adenosine minimal medium and the limited nutrition may act as a selection pressure to the resistance (Maeda et al., 2012). In support, are sent report using two competing strains of Pseudomonas aeruginosa showed that the development of QSIresistance is limited by two distinct factors. Firstly, development of resistance to QSIs is restricted when QS signaling is inadequate. Secondly, QSI-resistant bacteria are inhibited by social cheating of QSIsensitive bacteria (Gerdt and Blackwell, 2014). 
Additionally, most QSIs have a narrow spectrum of activity and are strain specific, significantly restricting the prevalence of resistant gene contributed by normal flora and other pathogens.

QSIs are well accepted as promising alternatives to antibiotics, but more experiments and time are needed regarding the effectiveness of these drugs and their tendency of inducing resistance.

\section{References}

Allen, R.C., R. Popat, S.P. Diggle and S.P. Brown, 2014. Targeting virulence: Can we make evolution-proof drugs? Nat. Rev. Microbiol., 12: 300-308. DOI: $10.1038 /$ nrmicro3232

Andersson, D.I. and D. Hughes, 2010. Antibiotic resistance and its cost: Is it possible to reverse resistance? Nat. Rev. Microbiol., 8: 260-271.

DOI: $10.1038 /$ nrmicro2319
Defoirdt, T., N. Boon and P. Bossier, 2010. Can bacteria evolve resistance to quorum sensing disruption? PLoS Pathogens, 6: e1000989-e1000989. DOI: 10.1371/journal.ppat.1000989

Gerdt, J.P. and H.E. Blackwell, 2014. Competition studies confirm two major barriers that can preclude the spread of resistance to quorum-sensing inhibitors in bacteria. ACS Chem. Biol., 9: 2291-2299.

DOI: $10.1021 / \mathrm{cb} 5004288$

Jiang, T. and M. Li, 2013. Quorum sensing inhibitors: A patent review. Expert Opin. Therapeutic Patents, 23: 867-894. DOI: 10.1517/13543776.2013.779674

Maeda, T., R. Garcia-Contreras, M. Pu, L. Sheng and L.R. Garcia et al., 2012. Quorum quenching quandary: Resistance to antivirulence compounds. ISME J., 16: 493-501. DOI: 10.1038/ismej.2011.122

Miller, M.B. and B.L. Bassler, 2001. Quorum sensing in bacteria. Annual Rev. microbial., 55: 165-199.

DOI: 10.1146/annurev.micro.55.1.165 